\title{
Therapeutic Effect of Direct Hemoperfusion with a Polymyxin B-Immobilized Fiber Column in the Treatment of HIV-Negative Severe Pneumocystis Pneumonia
}

\author{
Ryo Tachikawa ${ }^{a}$ Keisuke Tomii ${ }^{a}$ Kimihiko Murase $^{\mathrm{a}}$ Hiroyuki Ueda ${ }^{\mathrm{b}}$ \\ Yuka Harada ${ }^{e}$ Yoko Kida ${ }^{c}$ Kyosuke Ishihara ${ }^{\mathrm{d}}$ \\ Departments of ${ }^{\mathrm{a}}$ Respiratory Medicine and ${ }^{\mathrm{b}}$ Radiology, Kobe City Medical Center General Hospital, \\ 'Department of Respiratory Medicine, Kobe City Medical Center West Hospital, and ${ }^{\mathrm{d}}$ Kobe City Medical Center

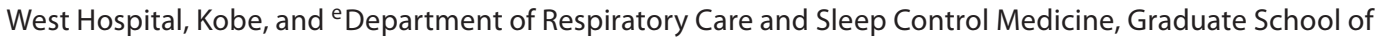 \\ Medicine, Kyoto University, Kyoto, Japan
}

\section{Key Words}

Adjunctive therapy - Diffuse alveolar damage •

Hemoperfusion • Pneumocystis pneumonia - Polymyxin B

\section{Abstract}

Background: Direct hemoperfusion with a polymyxin B-immobilized fiber column (PMX-DHP) has been shown to improve oxygenation in cases of diffuse alveolar damage, but little is known about its effectiveness in treating pneumocystis pneumonia (PCP) in HIV-negative immunosuppressed patients. Objectives: This study was aimed at investigating the effect of PMX-DHP in treating non-HIV-related PCP. Methods: Between October 2005 and September 2008, 6 patients with non-HIV-related PCP were treated with 2 sessions of PMX-DHP at an attending physician's discretion when severe hypoxemia developed despite conventional treatments including high-dose corticosteroid, whereas 9 patients in a similar condition were treated without PMXDHP. Changes in oxygenation and radiographic findings in the PMX-DHP group and adverse events associated with PMX-DHP were investigated retrospectively, as were the outcomes for both treatment groups. Results: There was an im- provement in $\mathrm{PaO}_{2} / \mathrm{FiO}_{2}$ during each $\mathrm{PMX}$-DHP session, from $148.8 \pm 52.5$ to $188.2 \pm 79.3 \mathrm{~mm} \mathrm{Hg}(p=0.02)$. After 2 sessions of PMX-DHP, an improvement in $\mathrm{PaO}_{2} / \mathrm{FiO}_{2}$, from 131.8 \pm 37.4 to $213.3 \pm 87.3 \mathrm{~mm} \mathrm{Hg}$, was observed $(p=0.04)$, but no significantly different improvement was detected on the following day. The radiographic findings improved in 4 patients during PMX-DHP. The in-hospital mortality was similarly high in both groups (50\% in the PMX-DHP group vs. $67 \%$ in the non-PMX-DHP group). No significant adverse events associated with PMX-DHP were observed except for advanced thrombocytopenia in 1 patient. Conclusion: PMXDHP may serve as an adjunct in the treatment of non-HIVrelated $\mathrm{PCP}$, temporarily alleviating severe hypoxemia even in cases refractory to conventional treatments.

Copyright $\odot 2010$ S. Karger AG, Basel

\section{Introduction}

Pneumocystis pneumonia (PCP) is a major cause of morbidity and mortality among immunocompromised individuals. Although PCP remains the leading AIDSdefining opportunistic infection throughout the world,

\section{KARGER}

Fax +41613061234

E-Mail karger@karger.ch

www.karger.com (c) 2010 S. Karger AG, Basel

$0025-7931 / 11 / 0814-0318 \$ 38.00 / 0$

Accessible online at:

www.karger.com/res
Ryo Tachikawa, MD

Department of Respiratory Medicine

Kobe City Medical Center General Hospital

4-6 Minatojima-nakamachi, Chuo-ku, Kobe 650-0046 (Japan)

Tel. +81 78302 4321, Fax +81 78302 2487, E-Mail ryotkw@ kcgh.gr.jp 
the introduction of highly active antiretroviral therapy and prophylaxis against PCP has resulted in a substantial decline in this disease among HIV-infected patients in industrialized countries. In contrast, the incidence of PCP in patients with predisposing immunodeficiency states other than HIV infection is increasing in frequency, further highlighting the clinical importance of nonHIV-related PCP $[1,2]$.

HIV-negative status is known to be associated with increased mortality from PCP compared with HIV-positive status [3-6]. Since inflammatory responses during PCP are more closely related to lung injury than the direct virulence of the organism, the relatively well-maintained immune function of HIV-negative patients provokes marked inflammatory responses, resulting in severe lung injury and respiratory impairment. As a result, HIV-negative patients typically present with an abrupt onset and a fulminant course of illness with a low organism burden, while HIV-infected patients experience a more insidious disease course with a larger organism burden.

Adjunctive corticosteroid has been studied as a way of palliating respiratory failure in the treatment of PCP. In HIV-infected patients, corticosteroids are of benefit in moderate to severe cases, reducing the inflammatory response and resulting in clinical improvement with reduced mortality [7]. On the other hand, the benefit of adjunctive corticosteroid has not been established in patients without HIV infection [8, 9]. Nevertheless, adjunctive corticosteroid is often essential in treating non-HIVrelated PCP to suppress exuberant inflammatory responses, especially in severe cases. However, the mortality of non-HIV-related PCP remains as high as $40 \%$ and reaches $60 \%$ in cases requiring ventilatory support $[1,6]$.

Direct hemoperfusion with a polymyxin B-immobilized fiber column (PMX-DHP) is an extracorporeal therapy originally developed for the treatment of sepsis, and it has proved to be effective in this fatal condition $[10,11]$. The rationale underlying this therapy is to remove circulating plasma endotoxin, thus preventing progression of the biological cascade of sepsis [12]. More recently, PMXDHP was reported to improve oxygenation in patients with acute lung injury or acute respiratory distress syndrome due to various causes, including sepsis $[13,14]$ and acute exacerbation of interstitial pneumonia $[15,16]$, which are pathologically characterized by diffuse alveolar damage. Similarly, diffuse alveolar damage is the most frequent pathologic finding in non-HIV-related PCP [5], but little is known about the efficacy of PMX-DHP in the management of this life-threatening opportunistic infection.
In this context, we have used PMX-DHP along with corticosteroid in severe cases of non-HIV-related PCP to ameliorate respiratory failure. To examine the effectiveness of PMX-DHP on non-HIV-related PCP, we retrospectively investigated the changes in oxygenation and radiographic findings in these patients, as well as the adverse events associated with PMX-DHP therapy. We also investigated the outcomes to assess the impact of PMXDHP on survival, compared with patients in the same condition who were treated without PMX-DHP.

\section{Patients and Methods}

\section{Patient Inclusion}

We conducted a retrospective review of 27 patients without HIV infection who had been given a diagnosis and treated for their PCP in our department between October 2005 and September 2008. We retrieved 20 cases of severe PCP defined as $\mathrm{PaO}_{2} /$ $\mathrm{FiO}_{2}$ (arterial partial pressure of oxygen/fraction of inspired oxygen) $<200 \mathrm{~mm}$ Hg during the treatment.

All patients presented with an abrupt onset of respiratory symptoms and bilateral interstitial infiltrates on chest CT, and bronchoscopy was performed in all but 1 case. Definitive diagnosis of PCP was made when pneumocystis cysts were confirmed in bronchoalveolar lavage fluid (BALF) by Grocott-Gomori methenamine-silver stain. If the specimen was negative for pneumocystis cysts, or was unavailable, clinical diagnosis was made when PCR for pneumocystis DNA was positive in BALF or sputum and the value of serum $\beta$-D-glucan was above normal. To exclude coexisting infections, blood, sputum and BALF cultures were performed, as was cytomegalovirus antigenemia assay. Cases which did not fulfill the definitive or clinical diagnosis criteria were excluded from the study, as were subjects suspected of having comorbid infection, congestive heart failure or pre-existing interstitial pneumonia. Fifteen cases were included in the study.

\section{PMX-DHP Therapy}

From October 2006, PMX-DHP (Toraymyxin 20R; Toray Medical Co., Tokyo, Japan) was considered as a treatment option for PCP when severe hypoxemia $\left(\mathrm{PaO}_{2} / \mathrm{FiO}_{2}<200 \mathrm{~mm} \mathrm{Hg}\right)$ developed despite conventional therapy. Each attending physician made the decision as to whether or when to use PMX-DHP therapy. Of the 15 patients included in the study, 6 were treated with PMX-DHP and 9 without.

PMX-DHP was administered through a double-lumen venous catheter for 4-24 h once daily for 2 successive days at a flow rate of $80-120 \mathrm{ml} / \mathrm{min}$. Nafamostat mesilate was used as an anticoagulant during PMX-DHP. $\mathrm{PaO}_{2} / \mathrm{FiO}_{2}$ was measured just before and after each PMX-DHP session with the same ventilator settings if used, as were other acute-phase parameters such as peripheral leukocytes, C-reactive protein and systolic blood pressure. A chest radiograph was taken before and after PMXDHP therapy, and radiographic changes were evaluated by a radiologist (H.U.), who was blinded to any clinical information. Plasma endotoxin was measured in all but 1 patient treated with PMX-DHP. 
Treatment for PCP

Before the results of the diagnostic examinations were obtained, presumptive therapy for PCP was initiated. Trimethoprim-sulfamethoxazole was the first choice of antibiotic, but this was replaced with pentamidine if continuing with this drug proved to be difficult due to its adverse effects. Antibiotic administration was continued for 14-21 days. Adjunctive corticosteroid therapy was initially administered as pulse therapy (methylprednisolone $500-1,000 \mathrm{mg} /$ day for 3 days), followed by a lower dose (methylprednisolone 40-125 mg/day), and was tapered off with improvement in respiratory status. Noninvasive ventilation (NIV) was, in principle, the first-line therapy for mechanical ventilation, but invasive ventilation was considered when NIV failed.

\section{Statistics and Ethics}

Continuous variables are expressed as means \pm SD and were compared between the groups by using Student's t test. Individual comparisons were performed with a 2 -sided paired t test. Categorical variables were compared using Fisher's exact test. Survival rates were expressed applying the Kaplan-Meier method, and the difference was assessed via a log-rank test. $p$ values $<0.05$ were considered statistically significant. All statistical analyses were performed using JMP 7.0.2 (SAS Institute Inc., Cary, N.C., USA) software. This study was approved by our institutional review board, and informed consent was obtained from patients or relatives in all cases treated with PMX-DHP.

\section{Results}

\section{Patient Characteristics}

No significant differences were seen in the clinical characteristics or laboratory data between the patients treated with or without PMX-DHP (table 1). In both groups, oxygenation was severely impaired, and serum $\mathrm{LDH}$ and neutrophil percentage in BALF were elevated. Most patients had malignant disease or received immunosuppressive agents, and all were considered to be immunocompromised. None of the patients received prophylaxis against PCP. Plasma endotoxin was negative in all cases measured.

\section{Treatments}

The treatments received and patient outcomes are summarized in table 2. Five patients in the PMX-DHP group and 6 in the non-PMX-DHP group required mechanical ventilation. Trimethoprim-sulfamethoxazole was replaced with pentamidine in 5 patients of both groups, mainly due to thrombocytopenia. PMX-DHP was performed within 1 week of the initiation of corticosteroid pulse therapy. PMX-DHP was administered to 4 patients after 3 days of corticosteroid pulse therapy, while 2 patients received PMX-DHP at least partly concomitantly with their corticosteroid pulse therapy.
Table 1. Baseline patient characteristics

\begin{tabular}{|c|c|c|}
\hline & $\begin{array}{l}\text { Patients treated } \\
\text { with PMX-DHP } \\
(\mathrm{n}=6)\end{array}$ & $\begin{array}{l}\text { Patients treated } \\
\text { without PMX- } \\
\text { DHP }(n=9)\end{array}$ \\
\hline Age, years & $57.3 \pm 17.8$ & $60.2 \pm 12.9$ \\
\hline Gender (male/female) & $4 / 2$ & $6 / 3$ \\
\hline \multicolumn{3}{|l|}{ Underlying diseases } \\
\hline Malignancy & 2 & 5 \\
\hline Posttransplant & 2 & 3 \\
\hline Other & 2 & 1 \\
\hline Duration of symptoms, days & $4.8 \pm 2.3$ & $7.7 \pm 6.3$ \\
\hline Immunosuppressive agents & 5 & 8 \\
\hline Prophylaxis & 0 & 0 \\
\hline Diagnosis (definitive/clinical) & $2 / 4$ & $3 / 6$ \\
\hline $\mathrm{PaO}_{2} / \mathrm{FiO}_{2}, \mathrm{~mm} \mathrm{Hg}$ & $167 \pm 49.2$ & $171 \pm 67.0$ \\
\hline $\mathrm{LDH}, \mathrm{U} / \mathrm{ml}$ & $587 \pm 189$ & $493 \pm 275$ \\
\hline$\beta$-D-glucan, pg/ml & $327 \pm 354$ & $96.6 \pm 90.8$ \\
\hline \multicolumn{3}{|l|}{ BALF } \\
\hline Neutrophil, \% & $31.0 \pm 29.1$ & $29.4 \pm 33.4$ \\
\hline Lymphocyte, \% & $31.8 \pm 27.5$ & $26.5 \pm 21.0$ \\
\hline
\end{tabular}

Figures are means \pm SD or numbers of cases.

\section{Effects of PMX-DHP}

All patients in the PMX-DHP group experienced progressive hypoxemia despite conventional treatment for PCP, and $\mathrm{PaO}_{2} / \mathrm{FiO}_{2}$ was $<200 \mathrm{~mm}$ Hg before PMX-DHP was performed. There was an improvement in $\mathrm{PaO}_{2} / \mathrm{FiO}_{2}$ during each PMX-DHP session, from $148.8 \pm 52.5 \mathrm{~mm}$ $\mathrm{Hg}$ (before PMX-DHP) to $188.2 \pm 79.3 \mathrm{~mm} \mathrm{Hg}$ (after PMX-DHP) $(\mathrm{p}=0.02)$. After 2 sessions of PMX-DHP, a significant improvement in $\mathrm{PaO}_{2} / \mathrm{FiO}_{2}$ compared to just before PMX-DHP therapy was observed (from $131.8 \pm$ 37.4 to $213.3 \pm 87.3 \mathrm{~mm} \mathrm{Hg}$; $=0.04$ ). However, no significant improvement was detected on the following day (fig. 1).

With regard to other acute-phase parameters, there were no significant changes in peripheral leukocytes (from $8,990 \pm 6,330$ to $6,500 \pm 3,560 / \mathrm{mm}^{3} ; \mathrm{p}=0.09$ ) or in C-reactive protein (from $10.0 \pm 10.3$ to $11.0 \pm 12.1$ $\mathrm{mg} / \mathrm{dl} ; \mathrm{p}=0.18$ ) during each PMX-DHP session, but a significant increase in systolic blood pressure was observed (from $124 \pm 16$ to $141 \pm 18 \mathrm{~mm} \mathrm{Hg}$; $\mathrm{p}=0.01$ ).

In the chest radiographs, bilateral infiltrates improved in 4 patients (patients 1, 2, 3 and 6) after 2 sessions of PMX-DHP therapy. However, 2 of them (patients 1 and 3) experienced deterioration in radiographic findings on the following day, accompanied by a deterioration in oxygenation. 


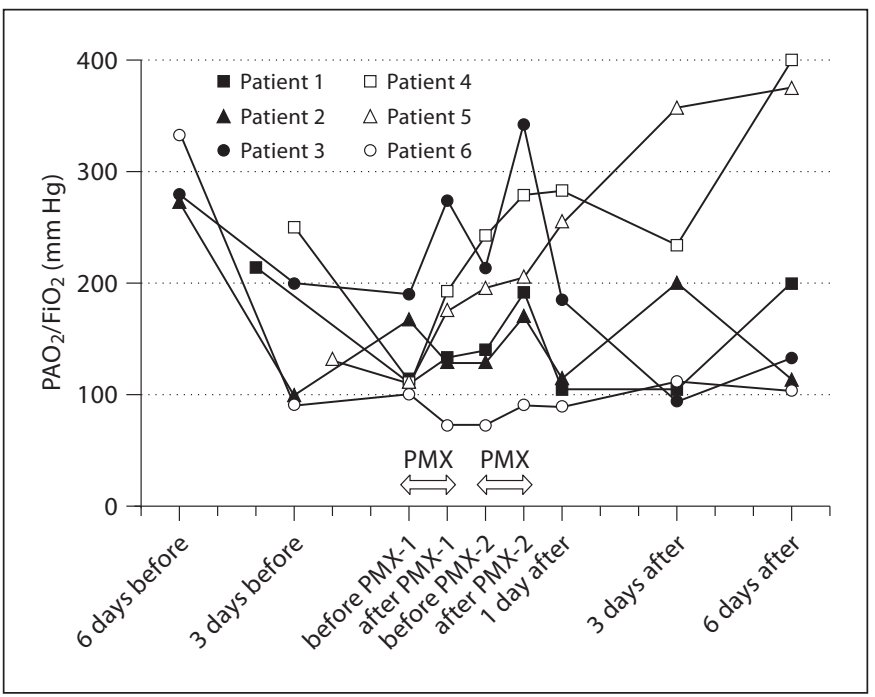

Fig. 1. Changes in $\mathrm{PaO}_{2} / \mathrm{FiO}_{2}$ in 6 patients with non-HIV-related pneumocystis pneumonia treated with 2 sessions of PMX-DHP (direct hemoperfusion with a polymyxin B-immobilized column) in addition to standard conventional therapy.

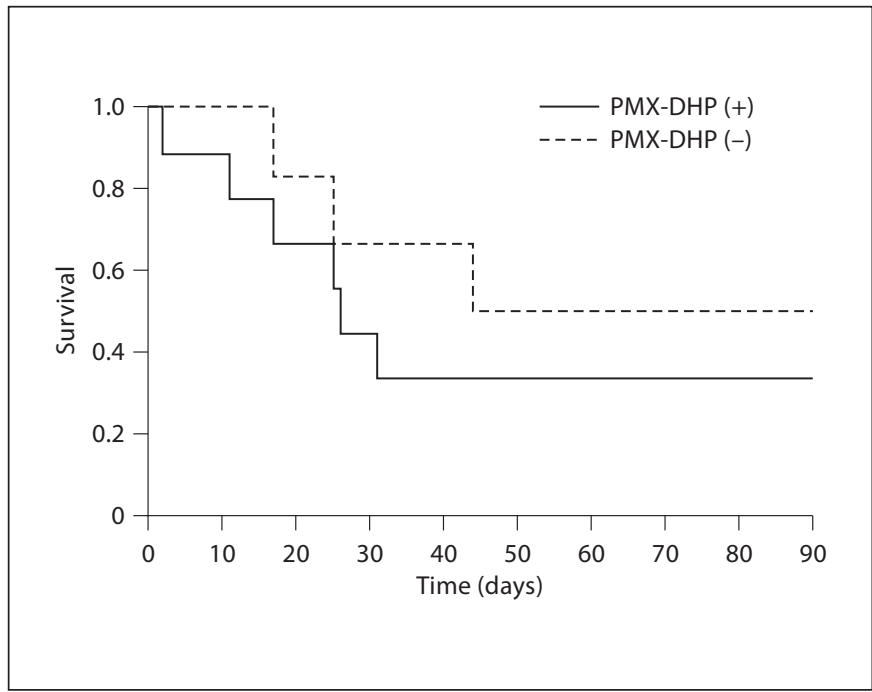

Fig. 2. Survival rates according to treatment group. Although no significant difference was found between the two groups ( $\mathrm{p}=$ 0.42 ), there was a trend toward a better survival in the PMX-DHP group.

Table 2. Summary of the treatments and outcomes

\begin{tabular}{|c|c|c|c|c|c|c|c|}
\hline & \multicolumn{2}{|l|}{ PMX-DHP } & $\begin{array}{l}\text { Mechanical } \\
\text { ventilation } \\
\text { (NIV) }\end{array}$ & \multicolumn{2}{|l|}{ Antibiotics } & $\begin{array}{l}\text { Outcome } \\
\text { (improved/ } \\
\text { died) }\end{array}$ & $\begin{array}{l}\text { Cause } \\
\text { of death } \\
\text { (PCP/ } \\
\text { others) }\end{array}$ \\
\hline PMX group $(n=6)$ & $0-6($ median 4$)$ & 4-24 (median 6) & $5(4)$ & 1 & 5 & $3 / 3$ & $0 / 3$ \\
\hline Non-PMX group $(n=9)$ & - & - & $6(5)$ & 4 & 5 & $3 / 6$ & $4 / 2$ \\
\hline
\end{tabular}

SMX-TMP = Trimethoprim-sulfamethoxazole.

\section{Outcome}

Three of six patients (50\%) in the PMX-DHP group died during their hospital stay, compared to 6 of $9(67 \%)$ in the non-PMX-DHP group. None of the patients in the PMX-DHP group died by day 14, whereas 2 patients (22\%) in the non-PMX-DHP group died during this period (fig. 2). The presumed principal cause of death in the PMX-DHP group was aspiration pneumonia (1 patient) or ventilator-associated pneumonia (2 patients). In the non-PMX-DHP group, the principal cause of death was presumed to be respiratory failure due to PCP (4 patients), ventilator-associated pneumonia (1 patient) or progression of underlying malignant disease (1 patient).

\section{Adverse Events of PMX-DHP}

In $83 \%(10 / 12)$ of the PMX-DHP sessions, peripheral blood platelets decreased by $27.5 \%$ (range: $4-65 \%$ ). The platelet levels recovered spontaneously in most patients, but 1 patient needed a platelet transfusion because of severe thrombocytopenia. There were no other significant adverse events associated with PMX-DHP therapy.

\section{Discussion}

In this retrospective study of severe non-HIV-related PCP, improvement was not observed with regard to inhospital mortality, but a temporal improvement in oxy- 
genation and radiographic findings during PMX-DHP treatment was observed without significant adverse events. To the best of our knowledge, this is the first report demonstrating the potential effectiveness of PMXDHP in the treatment of PCP as adjunctive therapy other than corticosteroid.

To date, the precise mechanism by which PMX-DHP improves oxygenation remains unknown. It is believed not to be associated with endotoxin removal because the beneficial effect is also observed in the treatment of grampositive bacterial infection $[13,14]$ or acute exacerbation of interstitial pneumonia $[15,16]$. Recent studies have reported that PMX-DHP reduces inflammatory mediators, which play an important role in diffuse alveolar damage, such as neutrophil elastase and IL-8 [14], metalloproteinase-9 [17] or neutrophil-reactive oxygen species [18], and the absorption of activated monocytes and neutrophils producing these mediators is speculated to be a possible treatment mechanism [19]. This provides a therapeutic rationale for using PMX-DHP in non-HIV-related PCP, which is characterized by neutrophilic lung inflammation that often results in diffuse alveolar damage. In the present study, a significant improvement in $\mathrm{PaO}_{2} / \mathrm{FiO}_{2}$ during PMX-DHP was observed without detectable endotoxins, which was well correlated with radiographic changes. This effect is thought to be one of the beneficial systemic influences of PMX-DHP, as is the observed hemodynamic improvement during the procedure; PMXDHP might improve oxygenation by reducing pulmonary vascular permeability and pulmonary edema by some mechanism other than endotoxin removal.

The nature of PMX-DHP therapy is shown by the finding that the improvement in oxygenation was not long lasting, probably because PMX-DHP does not directly address the source of infection and cannot afford a definitive cure. At the same time, however, our findings indicate that PMX-DHP, at least temporarily, alleviates respiratory insufficiency. In fact, no patients in the PMXDHP group died in the first 2 weeks of the treatment despite rapidly progressive respiratory failure, whereas $22 \%$ of the patients in the non-PMX-DHP group died during the same period. Considering that the PMX-DHP group was initially as severely affected as the non-PMXDHP group, or might even include more refractory cases because most of them did not respond to high-dose corticosteroid therapy, favorable survival in the early phase of the treatment could be attributed to the therapeutic effect of PMX-DHP. On the other hand, the in-hospital mortality in the PMX-DHP group was similar to that seen for the non-PMX-DHP group, and was as high as previously reported $[1,6]$. Further investigation will be needed to determine whether PMX-DHP can bring about a better prognosis in non-HIV-related PCP patients.

Importantly, the 3 deceased patients in the PMX-DHP group at one time showed signs of recovery and survived their initial respiratory insufficiency. However, they eventually all died of complications of pulmonary infection. This was in strong contrast to the non-PMX-DHP group, where most of the patients died of respiratory failure caused by PCP itself. This difference suggests that appropriate respiratory care plays a key role in determining the outcome. In this regard, NIV is recommended as a first-line therapeutic modality for respiratory failure among immunosuppressed patients as a means to avoid tracheal intubation and its associated poor prognosis [20]. However, severe respiratory impairment of nonHIV-related PCP often makes it difficult to continue NIV throughout the treatment and, more seriously, NIV failure is associated with a dismal prognosis [6]. Therefore, PMX-DHP therapy might serve as an adjunct that increases tolerance for NIV by alleviating respiratory failure, leading to a better prognosis.

Although 2 sessions of PMX-DHP for 2-3 h each have been used in the treatment of sepsis, it remains unclear what the optimal duration and the number of PMX-DHP sessions should be in order to improve oxygenation in cases associated with diffuse alveolar damage. Since recent studies have indicated that a longer duration may be more effective [16, 21], the duration of PMX-DHP was longer than usual in our patients, while the number of sessions was restricted to 2 . If longer or repeated PMXDHP therapy yields additional benefit, it will be more effective in alleviating respiratory failure in the treatment of non-HIV-related PCP.

Potential adverse events of PMX-DHP include thrombocytopenia and hypotension during treatment, but very few adverse events have been reported previously [10]. The treatment was also generally well tolerated in our patients, but 1 of them required platelet transfusion. Since thrombocytopenia tends to occur during the treatment of PCP due to adverse effects of trimethoprim-sulfamethoxazole dosing, care must be taken for patients with advanced thrombocytopenia.

Our study has several limitations. First, as this was a retrospective analysis and the patient number was small, it is open to selection bias and error. Second, the diagnosis of PCP was made largely based on PCR and serum $\beta$ D-glucan, probably due to the low sensitivity of microscopic BALF examination in HIV-negative patients. However, PCR and serum $\beta$-D-glucan are reliable mark- 
ers for the diagnosis of non-HIV-related PCP [22, 23], and almost all patients in the study underwent bronchoscopy to exclude concomitant infections. Thus, the patients included were considered to be infected only with pneumocystis. Finally, as corticosteroids were concurrently administered during PMX-DHP, the observed improvement in oxygenation may not accurately reflect the effect of PMX-DHP. Even so, initial corticosteroid pulse therapy was ineffective in most of the patients; thus the impact of corticosteroid on oxygenation was considered to be minimal. Indeed, some of the patients showed a dramatic improvement in oxygenation during PMX-DHP despite progressive respiratory deterioration with adjunctive corticosteroid.

\section{Conclusion}

PMX-DHP was safe and associated with a temporal improvement in respiratory status in severe non-HIV-related PCP patients, even when high-dose corticosteroid was ineffective. Our observations suggest a beneficial role for PMX-DHP in these patients as adjunctive therapy other than corticosteroid. A larger, prospective, randomized study is required to validate these results.

\section{Acknowledgements}

We thank Dr. Naohiro Toda, Dr. Koji Ueda, Dr. Yoshinori Taro and Dr. Akihiro Yoshimoto, Department of Nephrology, Kobe City Medical Center General Hospital, and Mr. Ichiro Sakaji and the other clinical engineers for administering PMX-DHP.

\section{References}

1 Mansharamani NG, Garland R, Delaney D, Koziel H: Management and outcome patterns for adult Pneumocystis carinii pneumonia, 1985 to 1995: comparison of HIV-associated cases to other immunocompromised states. Chest 2000;118:704-711.

2 Nuesch R, Bellini C, Zimmerli W: Pneumocystis carinii pneumonia in human immunodeficiency virus (HIV)-positive and HIVnegative immunocompromised patients. Clin Infect Dis 1999;29:1519-1523.

- 3 Limper AH, Offord KP, Smith TF, Martin WJ 2nd: Pneumocystis carinii pneumonia: differences in lung parasite number and inflammation in patients with and without AIDS. Am Rev Respir Dis 1989;140:12041209.

4 Curtis JR, Yarnold PR, Schwartz DN, Weinstein RA, Bennett CL: Improvements in outcomes of acute respiratory failure for patients with human immunodeficiency virus-related Pneumocystis carinii pneumonia. Am J Respir Crit Care Med 2000;162: 393-398.

5 Thomas CF Jr, Limper AH: Pneumocystis pneumonia. N Engl J Med 2004;350:24872498.

6 6 Monnet X, Vidal-PetiotE, Osman D, Hamzaoui O, Durrbach A, Goujard C, Miceli C, Bouree P, Richard C: Critical care management and outcome of severe pneumocystis pneumonia in patients with and without HIV infection. Crit Care 2008;12:R28.
$>7$

Bozzette SA, Sattler FR, Chiu J, Wu AW, Gluckstein D, Kemper C, Bartok A, Niosi J, Abramson I, Coffman J, et al, California Collaborative Treatment Group: A controlled trial of early adjunctive treatment with corticosteroids for Pneumocystis carinii pneumonia in the acquired immunodeficiency syndrome. N Engl J Med 1990;323:14511457.

8 Pareja JG, Garland R, Koziel H: Use of adjunctive corticosteroids in severe adult nonHIV Pneumocystis carinii pneumonia. Chest 1998;113:1215-1224.

\9 Delclaux C, Zahar JR, Amraoui G, Leleu G, Lebargy F, Brochard L, Schlemmer B, BrunBuisson C: Corticosteroids as adjunctive therapy for severe Pneumocystis carinii pneumonia in non-human immunodeficiency virus-infected patients: retrospective study of 31 patients. Clin Infect Dis 1999;29: 670-672.

10 Cruz DN, Perazella MA, Bellomo R, de Cal M, Polanco N, Corradi V, Lentini P, Nalesso F, Ueno T, Ranieri VM, Ronco C: Effectiveness of polymyxin B-immobilized fiber column in sepsis: a systematic review. Crit Care 2007;11:R47.

11 Cruz DN, Antonelli M, Fumagalli R, Foltran F, Brienza N, Donati A, Malcangi V, Petrini F, Volta G, Bobbio Pallavicini FM, Rottoli F, Giunta F, Ronco C: Early use of polymyxin B hemoperfusion in abdominal septic shock: the EUPHAS randomized controlled trial. JAMA 2009;301:2445-2452.

12 Shoji H: Extracorporeal endotoxin removal for the treatment of sepsis: endotoxin adsorption cartridge (Toraymyxin). Ther Apher Dial 2003;7:108-114.
13 Tsushima K, Kubo K, Koizumi T, Yamamoto H, Fujimoto K, Hora K, Kan-Nou Y: Direct hemoperfusion using a polymyxin B immobilized column improves acute respiratory distress syndrome. J Clin Apher 2002;17:97102

14 Kushi H, Miki T, Okamaoto K, Nakahara J, Saito T, Tanjoh: Early hemoperfusion with an immobilized polymyxin B fiber column eliminates humoral mediators and improves pulmonary oxygenation. Crit Care 2005; 9:R653-R661.

15 Seo Y, Abe S, Kurahara M, Okada D, Saito Y, Usuki J, Azuma A, Koizumi K, Kudoh S: Beneficial effect of polymyxin B-immobilized fiber column (PMX) hemoperfusion treatment on acute exacerbation of idiopathic pulmonary fibrosis. Intern Med 2006;45: 1033-1038.

16 Enomoto N, Suda T, Uto T, Kato M, Kaida Y, Ozawa Y, Miyazaki H, Kuroishi S, Hashimoto D, Naito T, Fujisawa T, Matsui T, Inui N, Nakamura Y, Sato J, Mizuguchi T, Kato A, Chida K: Possible therapeutic effect of direct haemoperfusion with a polymyxin B immobilized fibre column (PMX-DHP) on pulmonary oxygenation in acute exacerbations of interstitial pneumonia. Respirology 2008; 13 : 452-460.

17 Nakamura T, Kawagoe Y, Matsuda T, Shoji H, Ueda Y, Tamura N, Ebihara I, Koide H: Effect of polymyxin B-immobilized fiber on blood metalloproteinase- 9 and tissue inhibitor of metalloproteinase-1 levels in acute respiratory distress syndrome patients. Blood Purif 2004;22:256-260. 
18 Naka T, Shinozaki M, Akizawa T, Shima Y, Takaesu H, Nasu H: The effect of continuous veno-venous hemofiltration or direct hemoperfusion with polymyxin B-immobilized fiber on neutrophil respiratory oxidative burst in patients with sepsis and septic shock. Ther Apher Dial 2006;10:7-11.

19 Tsujimoto H, Ono S, Hiraki S, Majima T, Kawarabayashi N, Sugasawa H, Kinoshita M, Hiraide H, Mochizuki H: Hemoperfusion with polymyxin B-immobilized fibers reduced the number of CD16+ CD14+ monocytes in patients with septic shock. J Endotoxin Res 2004;10:229-237.
20 Hilbert G, Gruson D, Vargas F, Valentino R, Gbikpi-Benissan G, Dupon M, Reiffers J, Cardinaud JP: Noninvasive ventilation in immunosuppressed patients with pulmonary infiltrates, fever, and acute respiratory failure. N Engl J Med 2001;344:481-487.

-21 Mitaka C, Tsuchida N, Kawada K, Nakajima Y, Imai T, Sasaki S: A longer duration of polymyxin B-immobilized fiber column hemoperfusion improves pulmonary oxygenation in patients with septic shock. Shock 2009;32: $478-483$.
2 Azoulay E, Bergeron A, Chevret S, Bele N, Schlemmer B, Menotti J: Polymerase chain reaction for diagnosing pneumocystis pneumonia in non-HIV immunocompromised patients with pulmonary infiltrates. Chest 2009; 135:655-661

23 Tasaka S, Hasegawa N, Kobayashi S, Yamada W, Nishimura T, Takeuchi T, Ishizaka A: Serum indicators for the diagnosis of pneumocystis pneumonia. Chest 2007;131:11731180. 